Canadian Oncology

Nursing Journal

Revue canadienne

de soins infirmiers

en oncologie

Volume 27, Issue 3 • Summer 2017

elSSN: $2368-8076$ 


\section{Dépistage systématique de la détresse : portraits et regards croisés sur les perceptions des différents acteurs, perspectives cliniques et de recherche- Partie I}

par Nicole Tremblay, Odette Roy, Aurélie Lecocq, avec la collaboration de Caroline Provencher, Louise Compagna, Claudine Tremblay, Francine Grondin et Karine Le Breton et des assistantes de recherche Jocelyne Doucet et Cécile Mathurin

Cet article présente les résultats ayant trait à la première phase d'une étude à devis mixte séquentiel exploratoire (Creswell et Plano Clarke, 2007). Cette recherche a été rendue possible grâce au soutien financier de l'Association canadienne des infirmières en oncologie et de la Fondation de l'hôpital Maisonneuve-Rosemont, fonds administré par le Centre d'excellence en soins infirmiers de cette installation.

\section{INTRODUCTION}

$\mathrm{Q}$ uiconque côtoie une personne atteinte de cancer réalise rapidement que cette maladie impose de nombreux défis d'adaptation tant à elle qu'à ses proches aidants, et ce, tout au long de la trajectoire de soins et de traitements (Bultz et Carlson, 2006; Fitch, Howell, McLeod et Green, 2012). D'ailleurs, la littérature scientifique à ce sujet indique que de 35 à $45 \%$ des personnes atteintes de cancer vivent avec un certain degré de détresse (Carlson, Angen, Cullum, Goodey, Koopmans, Lamont et al., 2004; Mitchell, Hussain, Grainger et Symonds, 2011; Zabora, Brintzenhofeszoc, Curbow, Hooker et Piantadosi, 2001). La détresse varie notamment selon le type de cancer, le temps écoulé depuis le diagnostic et le stade de la maladie (Vachon, 2006). Plusieurs autres facteurs sont

\section{AU SUJET DES AUTEURES}

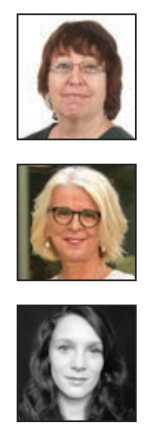

Nicole Tremblay, inf., M.Sc, CSIO(C), ICSP(C), Chercheure principale

Odette Roy, inf., M.Sc., M.A.P., Ph.D. Co-chercheure

Aurélie Lecocq, Ph.D. en éducation, psychométricienne chez Assessment strategies inc

Avec la collaboration de:

Caroline Provencher, inf., M.Sc.

Louise Compagna, inf., B.Sc.

Claudine Tremblay, inf., M.Sc., CSIO(C)

Francine Grondin, inf., B.Sc.

Karine Le Breton, inf., M.Sc., CSIO(C)

Et des assistantes de recherche:

Jocelyne Doucet, inf., B.Sc.

Cécile Mathurin, inf., M.Sc.

DOI: $10.5737 / 23688076211223$ présents : l'âge, le soutien social, les antécédents médicaux, les styles d'adaptation et autres variables personnelles (Weisman, 1976; Folkman et Greer, 2000 cités dans Fitch, Howell, McLeod et Green, 2012; NCCN, 2012) influencent aussi le niveau de détresse. De plus, certaines sous-populations, notamment les femmes atteintes de cancer du sein et les personnes greffées pour un cancer hématologique, seraient reconnues comme vivant davantage de détresse.

Ainsi, un patient sur deux expérimente de la détresse ( $\mathrm{N}=51)$ lorsqu'il est vu à sa première consultation pour une greffe de cellules hématopoïétiques (Trask, Paterson, Riba, Brines, Grifith, Parker et al., 2002). Les survivants ne sont pas épargnés. Ils font également face à de grands défis, comme l'illustre une analyse s'intéressant à la mortalité tardive chez des survivants de greffe de cellules hématopoïétiques ( $\mathrm{N}=1479)$. Dans cette étude, Bathia et ses collaborateurs rapportent que les greffés «long terme » (suivi médian de 9,5 ans) ont 14 fois plus de problèmes de santé qui les empêchent d'occuper un emploi comparativement à leurs frères et sœurs du même groupe d'âge. Ces défis influencent leur qualité de vie et leur bien-être fonctionnel (Bathia et al., 2007). De surcroît, les auteurs indiquent que $22 \%$ des survivants «long terme » souffrent de troubles psychologiques tels que l'anxiété, la dépression et la somatisation. Sept pour cent d'entre eux expriment des idéations suicidaires (Sun, Francisco, Baker, Weisdorf, Forman et Bathia, 2011). Une autre étude parue en 2008 indique que $43 \%$ des survivants à long terme éprouvent de la détresse psychologique (Rusiewicz, DuHamel, Burkhalter, Ostroff, Winkel, Scigliano et al., 2008).

De la même manière, les femmes touchées par le cancer du sein constituent un autre groupe particulièrement vulnérable à la détresse tout au long du continuum de soins (Groenvold, Petersen, Idler, Bjorner, Fayers et Mouridsen, 2007; Grunfeld, Coyle, Whelan, Clinch, Reyno, Earle et al., 2004). Ainsi, selon des études, elles vivent, autour de la période du diagnostic, une détresse exigeant une attention toute particulière dans 30 à $40 \%$ des cas (Compas et Luecken, 2002; Howard-Anderson, Ganz, Bower et Stanton, 2011). Par ailleurs, une étude citée par Vachon (2006) et réalisée auprès de survivantes long terme de cancer du sein indique que $18 \%$ des participantes rencontrent les critères du syndrome de stress post-traumatique et que plus de la moitié (58\%) présentent 2 des 3 groupes de symptômes de stress post-traumatique (Amir et Ramati, 2002, cités dans Vachon, 2006). Ces données revêtent un caractère important lorsque l'on sait que les femmes qui ont été traitées 
pour un cancer du sein représentent, avec les hommes atteints du cancer de la prostate, le plus grand groupe de survivants du cancer (Société canadienne du cancer, 2013).

Selon ce résumé non exhaustif d'une large littérature scientifique, la détresse représente un problème prévalent et important sur le plan des conséquences pour les femmes atteintes de cancer du sein et la population atteinte de cancer hématologique ayant eu la greffe comme modalité thérapeutique. Cela justifie la nécessité d'une étude portant sur la détresse vécue par la clientèle traitée dans notre établissement. En plus, de nombreuses observations cliniques répétées dans le temps et réalisées par les différents membres de notre équipe de recherche laissent entrevoir que l'anxiété constitue un symptôme prévalent chez ces deux clientèles bien distinctes, qui pourrait vraisemblablement expliquer en partie la détresse décrite antérieurement.

À cet égard, diverses études confirment la prévalence de l'anxiété chez ces populations, quoique ces études soient nettement plus nombreuses pour la population de cancer du sein que pour la population de cancer hématologique greffée. L'incidence plutôt faible des cancers hématologiques, la faible indication de la greffe de cellules hématopoiétiques comme traitement, et la mortalité élevée chez cette population peuvent expliquer en grande partie ce dernier constat (Niess et Duffy, 2004; Société canadienne du cancer, 2013¹). Nous constatons toutefois que peu de chercheurs en sciences infirmières ont exploré les facteurs qui contribuent à l'anxiété et à la détresse vécues par ces patients à partir d'un outil de dépistage systématique complété périodiquement. Ces données sont pourtant particulièrement importantes pour le développement de la pratique infirmière et d'une offre de services adaptée. Ceci justifie notre intérêt à approfondir notre compréhension de la détresse auprès des deux populations ciblées, par le biais d'une étude explorant les différentes facettes de l'anxiété, mais aussi d'autres aspects du dépistage, notamment les aspects relationnels (qui seront traités dans un deuxième article).

\section{LE CONTEXTE DU DÉPISTAGE SYSTÉMATIQUE DE LA DÉTRESSE DANS NOTRE ÉTABLISSEMENT}

Le Partenariat canadien contre le cancer (PCCC) et l'Association canadienne d'oncologie psychosociale (ACOP) ont émis, en 2009, des recommandations concernant l'évaluation des besoins en soins psychosociaux du patient adulte atteint de cancer. Ainsi, le dépistage de la détresse est recommandé comme premier indicateur - ou signal d'alarme - des besoins en soins psychosociaux. De même, Agrément Canada considère la détresse émotionnelle comme le $6^{\mathrm{e}}$ signe vital, après la tension artérielle, la température, le pouls, la respiration et la douleur, et l'a incorporée en tant que norme de soins au

1. Les cancers hématologiques (tous sites confondus) constituent le $5^{\mathrm{e}}$ cancer sur le plan de l'incidence. Bien que la survie se soit nettement améliorée dans les dernières années, il demeure que le pourcentage de survie relative à 5 ans est de $67 \%$ pour le lymphome et de $58 \%$ pour la leucémie, comparativement à $88 \%$ pour le cancer du sein. sein de son programme Qmentum « Services de traitement du cancer et oncologie » (Fitch, Howell, McLeod et Green, 2012; Programme Qmentum, 2010).

De fait, dans le but premier de mieux répondre aux besoins psychosociaux des personnes traitées pour un cancer à l'hôpital Maisonneuve-Rosemont (HMR), nous avons implanté en janvier 2012 un outil de dépistage systématique de la détresse. En effet, le Programme-clientèle d'oncologie de l'HMR avait ciblé à cette période trois sites pilotes pour l'implantation de l'outil, soit la clinique ambulatoire d'oncologie, le centre de référence pour investigation désigné pour le cancer du sein (CRID) et l'unité des soins palliatifs. Puis, le dépistage a été étendu à l'unité d'hospitalisation des cancers hématologiques en avril 2013, et à la greffe en mode externe ainsi qu'en radio-oncologie pour la clientèle avec cancer du sein en septembre de la même année. En juillet 2013, nous avons révisé les temps d'administration de l'outil de dépistage pour le centre d'oncologie et le CRID. Par ailleurs, une version identique aux autres secteurs a été implantée en soins palliatifs autour d'octobre 2013.

À l'été 2012, un groupe de travail s'est penché sur les résultats obtenus dans le cadre de l'implantation de l'outil sur les unités visées ci-dessus. À cette époque, des données préliminaires indiquaient que les aspects interactionnels et contextuels étaient déterminants pour la pratique clinique. Nous avons donc souhaité non seulement collecter des données liées au déploiement de cet outil auprès des clientèles de ces unités, mais également obtenir des informations auprès des infirmières et des patients en regard des enjeux d'ordre relationnel et organisationnel influençant l'implantation du dépistage systématique de la détresse en oncologie.

\section{MÉTHODOLOGIE}

Devis

Un devis mixte séquentiel exploratoire a été développé et utilisé afin de capturer des réalités complexes, telles que des interactions infirmières-patients portant sur un sujet pouvant être chargé émotivement, dans un contexte nouveau et imprévisible (Foss et Ellefsen, 2002).

\section{Objectifs de l'étude}

Cette étude poursuit cinq objectifs distincts. Comme il a été mentionné plus tôt, cet article présente les résultats ayant trait à la première phase de l'étude, soit le premier objectif, tandis qu'un deuxième article présentera les résultats ayant trait aux objectifs 3 et 5 . Notons que le $4^{\mathrm{e}}$ objectif sera abordé dans la dernière phase de l'étude, qui sera complétée en 2017. Les objectifs ont été écrits en se fondant sur les populations à l'étude. Les trois premiers sont identiques pour les deux clientèles atteintes de cancer. Le quatrième vise spécifiquement la population de cancers hématologiques et de greffe, tandis que le cinquième vise la population de cancer du sein.

\section{Populations de cancers hématologiques et de cancer du sein} Les objectifs sont de :

1. Recueillir des informations sur la détresse vécue par la clientèle ciblée à des moments précis de la trajectoire de soins; (phase 1) 
2. Collecter des informations sur le processus d'implantation du dépistage systématique de la détresse au sein des différents secteurs touchés par le dépistage; (phases 1 à 3)

3. Mieux connaître les perceptions infirmières à l'égard du dépistage systématique de la détresse en contexte de cancers hématologiques, de greffe de cellules hématopoiétiques et de cancer du sein; (phase 2)

Le $4^{e}$ objectif a été décrit ainsi :

4. Mieux connaître la perspective du patient à l'égard du dépistage systématique de la détresse en contexte de cancers hématologiques et de greffe de cellules hématopoiétiques; (phase 2 et 3 )

Tandis que le $5^{\mathrm{e}}$ est formulé comme suit :

5. Mieux comprendre les perceptions de la femme atteinte de cancer du sein à l'égard du dépistage de la détresse. (phase 2)

Ces deux derniers objectifs, bien que semblables, sont distincts sur le plan de la nature des données. Nous avons questionné la clientèle greffée par le biais d'outils quantitatifs (questionnaires), mais nous projetons le faire également à l'aide d'outils qualitatifs. Ces deux méthodes de collecte d'information sont complémentaires dans la mesure où les questionnaires permettent de mieux connaître les perceptions; une entrevue avec questions ouvertes permet au participant d'exprimer plus librement son point de vue. De surcroît, les résultats de la phase 1 et 2 de notre étude nous incitent à poursuivre en ce sens. Ces perceptions constituent donc le cœur des deux articles.

\section{Considérations éthiques}

Cette étude a obtenu, en début d'année 2013, un certificat éthique de l'établissement où elle s'est déroulée. Il est important de noter que le dépistage systématique de la détresse étant considérée comme une intervention standard en oncologie, il n'y a pas de consentement autre que l'accord verbal de la clientèle au moment du dépistage. Toutefois, un consentement écrit a été obtenu de la part des participants pour toutes les autres phases de l'étude.

\section{COLLECTE DE DONNÉES ET ANALYSES}

\section{Description des outils de collecte de données de la phase 1}

Les outils décrits dans les prochains paragraphes ont permis d'atteindre le but 1 de l'étude, qui est de recueillir des informations sur la détresse vécue par la clientèle ciblée à des moments précis de la trajectoire de soins.

\section{Outil de Dépistage de la Détresse (ODD)}

Loutil de dépistage de la détresse (ODD) choisi à l'HMR est le même instrument que celui utilisé par le Centre hospitalier universitaire de Québec (CHUQ) et pour lequel nous avons obtenu les autorisations d'adaptation et de reproduction. Cet outil répond aux considérations préconisées dans le «Guide pancanadien de pratique clinique : évaluation des besoins psychosociaux du patient adulte atteint de cancer » (2009).

Il comporte trois mesures distinctes. En premier lieu, le thermomètre de la détresse (TD) mesure un aspect (la détresse psychologique) à l'aide d'une échelle à 11 points ( 0 à 10$)$. Il permet au patient d'indiquer le niveau moyen de détresse de la dernière semaine (incluant la journée même).

Le TD a été validé dans différentes études auprès de différents groupes de patients atteints de cancer et notamment sur une unité de greffe de moelle osseuse (NCCN, 2012). Par ailleurs, un seuil clinique de référence de 5 a été choisi. Il s'agit du seuil qui conduit à une évaluation plus poussée et à une offre possible d'orientation vers un autre intervenant. C'est le seuil qui a été choisi également par l'équipe du CHUQ et qui représentait le meilleur équilibre entre sensibilité et spécificité pour une orientation vers un intervenant psychosocial (communication personnelle, De Serres, CHUQ).

En deuxième lieu, une liste canadienne de vérification des problèmes (LCVP) accompagne le thermomètre de détresse. L'usager coche, dans la liste, ce qui suscite une difficulté. Ces difficultés sont regroupées en six catégories ou domaines. Ces domaines sont ceux utilisés dans le cadre de soins de soutien aux personnes atteintes de cancer, qui sert de référence à la démarche d'implantation de l'outil de dépistage de la détresse (Fitch, 2008). Dans cette liste canadienne, le domaine psychologique est compris dans le domaine émotionnel. Cette liste est celle proposée dans le guide d'implantation du dépistage de la détresse, le $6^{\mathrm{e}}$ signe vital (PCCC, 2009). Nous avons ajouté un élément à cette liste dans le domaine social et familial : celui de vivre l'isolement de protection, car nous avons considéré qu'aucun élément de cette liste ne renvoyait à cette dimension propre de l'expérience de greffe de cellules hématopoiétiques qu'est l'isolement neutropénique sur une période prolongée.

Enfin, l'échelle d'évaluation des symptômes d'Edmonton (ESAS) permet au patient de noter, sur une échelle visuelle analogue allant de 0 à 10, l'intensité de dix symptômes (dont un au choix) au cours des dernières $24 \mathrm{~h}$. L'ESAS est un outil fiable sur le plan psychométrique et a été validé auprès de différentes populations, dont celle de patients atteints de cancer en phase avancée et celle des patients aux premières étapes de leur cheminement (PCCC, 2009).

Nous avons également choisi un seuil clinique de référence à 5 pour ce qui est de l'anxiété et de la dépression, seuil où il apparaît indispensable de procéder à une évaluation en psycho-oncologie et qui, encore une fois, est le même que celui utilisé par le CHUQ. Néanmoins, le jugement clinique posé sur une situation donnée peut être différent de ce que la conduite prédéterminée préconise. Par exemple, une anxiété rapportée à l'ODD peut être excessivement élevée avant une rencontre médicale. Dans un tel cas, l'offre d'une référence pourrait être différée jusqu'à une réévaluation de l'anxiété après la rencontre médicale. En ce cas, l'offre de référence demeure toujours du ressort de l'intervenant. Et d'ailleurs, comme nous le verrons, certains résultats témoignent de cette réalité clinique.

L'outil de dépistage utilisé permet aussi au patient d'indiquer le désir ou non de recevoir de l'aide pour les problèmes identifiés. Le professionnel revoit ensuite avec la personne les réponses au questionnaire complété et, selon les résultats, intervient de manière individualisée afin de favoriser le soulagement des symptômes ou de la détresse. 
Un formulaire de suivi de l'outil de dépistage permet à l'infirmière ou à tout autre professionnel procédant au dépistage et qui fait le retour de noter les interventions proposées au patient suite aux résultats. Sur ce formulaire, certaines informations médicales (diagnostic, stade de cancer) sont requises, et la situation du patient dans la trajectoire est questionnée. Un quatrième outil, la «trajectoire globale de réponse à la détresse », guide la référence aux différents professionnels en matière de soutien psychosocial au sein du programme (voir figure ci-après).

\section{Temps de mesure de la détresse}

La mesure 1 correspond, pour les patientes atteintes de cancer du sein, au $3^{e}$ rendez-vous médical, celui où se décide en général le plan de traitement, alors que pour les patients atteints de cancer hématologique, il correspond, pour la plupart d'eux, à l'admission du patient à l'unité de soins $\mathrm{ou}$, de manière plus rare, au $\mathrm{J} 1 \mathrm{du} 2^{\mathrm{e}}$ cycle de traitement de chimiothérapie.

La mesure 2 sera, pour les femmes atteintes de cancer du sein, soit le J1 du $2^{\text {e }}$ cycle de traitement de chimiothérapie, la $3^{e}$ semaine de traitement de radiothérapie, ou lors de la classe d'enseignement de préchirurgie. Pour les patients atteints de cancer hématologique, ce moment correspond au congé du patient ou, de manière plus rare, à une réadmission si le formulaire n'a pas été rempli au moment de donner le congé.

Les mesures 3 et 4 correspondent à un ODD réalisé dans le cadre d'un suivi post-greffe, en cours de traitement pour les deux populations choisies, ou bien à un ODD réalisé aux soins palliatifs.

Précisons ici que l'ensemble des analyses statistiques a été réalisé sous SPSS.

\section{RÉSULTATS}

\section{Caractéristiques des échantillons}

Au total, entre le $1^{\text {er }}$ août 2013 et le 31 août 2014, 582 questionnaires « Outil de dépistage de la détresse » (ODD) ont été distribués au premier temps de mesure (T1). Sur ces 582 questionnaires, 50 patients (soit 8,6\%) n'ont pas souhaité répondre. Au temps 2 (T2), nous avons obtenu 151 ODD complétés sur une possibilité de 532. Ce faible taux de réponse s'explique par une difficulté à systématiser le processus de dépistage de la détresse à d'autres moments que le temps 1 . Sur les 151 répondants du temps de mesure 2, 52 étaient des hommes (soit $34 \%$ ) et 99 étaient des femmes (soit $66 \%$ ).
Nous avons exclu les temps 3 et 4 de toutes les analyses en raison du peu de questionnaires complétés (42 et 10, respectivement). Par ailleurs, le portrait de la détresse brossé dans cet article porte principalement sur les résultats du T1, soit sur les 532 outils complétés à ce moment. Quant à la variation de la détresse, aux seuils de référence et à la réponse au besoin dans le temps, ils s'appuient sur les 151 ODD du temps 2.

Par ailleurs, 505 formulaires de suivi de l'outil de dépistage ont été remplis par les infirmières sur une possibilité de 532 (228 pour les participants avec cancers hématologiques et 277 pour les participantes avec cancer du sein). Le nombre de données valides est spécifié chaque fois.

Le tableau 1 présente les participants à l'étude selon le type de cancer des répondants et des non-répondants.

Les raisons relatives au refus de participation ont été recueillies, mais certains patients n'ont pas souhaité la mentionner. Pour le temps 1, 24 des 50 patients qui ont refusé de participer à l'étude ont justifié leur refus. Parmi eux, 18 femmes atteintes de cancer du sein ont répondu qu'elles n'en ressentaient pas le besoin. Les autres raisons invoquées sont, par ordre croissant, l'incapacité de répondre $(\mathrm{N}=3)$, la non-compréhension $\mathrm{du}$ français $(\mathrm{N}=2)$ et un désir de ne pas parler de ses émotions $(\mathrm{N}=1)$. Les analyses statistiques $\left(\mathrm{khi}^{2} \mathrm{et} \mathrm{t}\right.$-test) ont montré que rien ne distingue ces non-participants des participants en ce qui concerne l'âge, le genre ou le diagnostic.

L'âge moyen de l'ensemble des participants est de 58 ans au temps 1 , avec un minimum de 18 ans et un maximum de 95 ans, l'écart-type étant de 14 ans. L'âge moyen des participants au temps 2 est de 54 ans, avec un minimum de 22 ans et un maximum de 85 ans, l'écart-type étant de 13,5 ans. Les patientes atteintes de cancer du sein sont significativement plus âgées que les patients atteints de cancer hématologique au temps 1. En moyenne, les patientes atteintes de cancer du sein sont âgées de 62 ans (écart-type : 12,3), soit 10 ans de plus que les patients atteints de cancer hématologique, dont l'âge moyen est de 52 ans (écart-type : 13,7). Les résultats du t-test indiquent que cette différence est significative au temps 1 $(\mathrm{t}=-8,8 ; \mathrm{p}=0,000)$ et au temps $2(\mathrm{t}=-4,4 ; \mathrm{p}=0,000)$.

La figure 1 représente les différents diagnostics pour les participants cancers hématologiques. Cette donnée est disponible pour 228 participants. La leucémie représente le diagnostic le plus prévalent ( $39 \%)$, suivi des lymphomes non hodgkiniens $(27 \%)$.

La donnée relative au stade du cancer est disponible pour 489 participants (donnée inscrite dans le formulaire de suivi de l'outil de dépistage). Les infirmières ont inscrit « sans objet»

\begin{tabular}{|c|c|c|c|c|c|c|}
\hline & \multicolumn{3}{|c|}{$\mathrm{T} 1$} & \multirow{2}{*}{$\begin{array}{l}\text { Total T1 } \\
\text { valides }\end{array}$} & \multirow{2}{*}{$\begin{array}{c}\text { Total T1 } \\
\text { distribués }\end{array}$} & \multirow{2}{*}{$\begin{array}{l}\text { Total T2 } \\
\text { valides }\end{array}$} \\
\hline & Non complétés & Hommes & Femmes & & & \\
\hline \multicolumn{7}{|l|}{ Cancer } \\
\hline Hématologique & 14 & 151 & 92 & 243 & 257 & 83 \\
\hline Sein & 36 & & 289 & 289 & 325 & 68 \\
\hline Total & 50 & & & 532 & 582 & \\
\hline
\end{tabular}




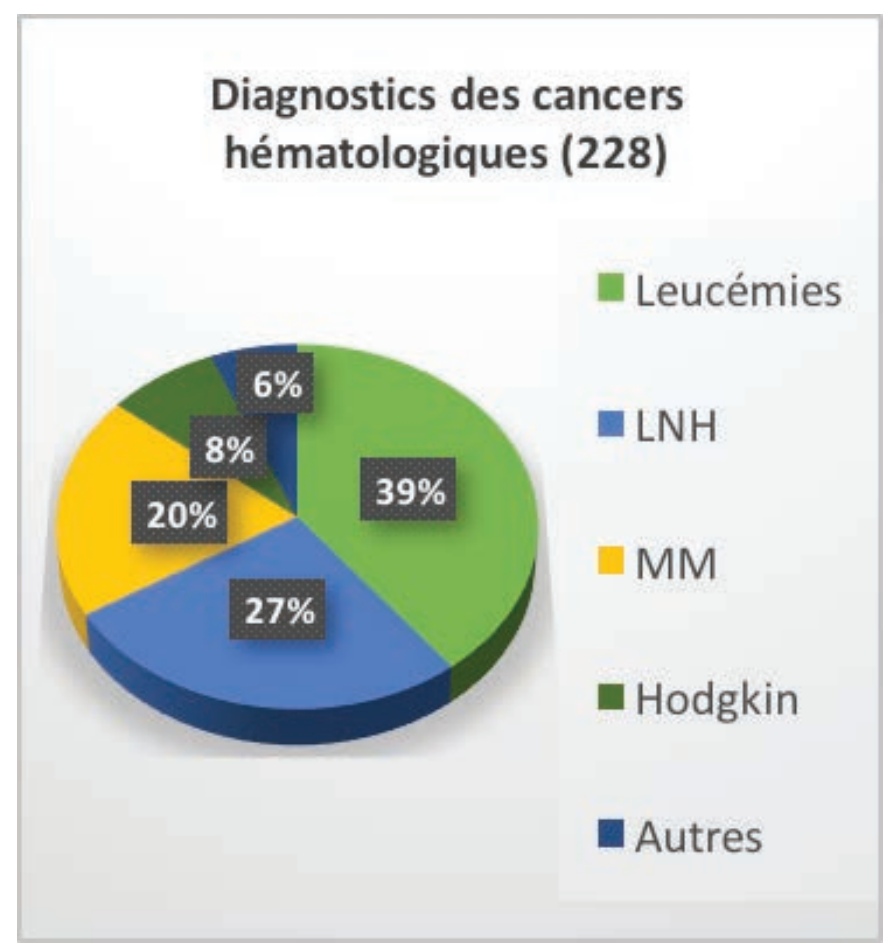

Figure 1. Diagnostics des cancers hématologiques

\begin{tabular}{|c|c|c|c|}
\hline \multicolumn{4}{|c|}{$\begin{array}{l}\text { Tableaux } 2 \mathrm{a} \text { et } 2 \mathrm{~b} \text { : Cote au thermomètre au temps } 1 \text {, selon le } \\
\text { genre et le type de cancer }\end{array}$} \\
\hline Genre & $\mathbf{N}$ & Moyenne & Écart-type \\
\hline Homme & 136 & 2,9 & 2,4 \\
\hline Femme & 374 & 3,3 & 2,8 \\
\hline Total & 510 & 3,2 & 2,7 \\
\hline
\end{tabular}

\begin{tabular}{|l|l|l|l|}
\hline Cancer & N & Moyenne & Écart-type \\
\hline Hémato & 225 & 3,2 & 2,5 \\
\hline Sein & 285 & 3,3 & 2,8 \\
\hline Total & 510 & 3,2 & 2,7 \\
\hline
\end{tabular}

$47 \%$ du temps (ce qui s'explique par l'échantillon avec cancers hématologiques, pour lequel le stade de cancer est sans objet). Si on exclut ce choix, $62 \%$ de l'échantillon avec cancer du sein était à un stade locorégional, $18 \%$ à un stade local, et un autre $18 \%$ à un stade métastatique.

\section{La cote au thermomètre de la détresse}

Le niveau moyen de détresse au temps 1 est de 3,2 (minimum de 0 , maximum de 10 , écart-type de 2,73). Au temps 2 , le niveau moyen de détresse est de 2,84 (é.-t. = 2,7). En moyenne, le niveau de détresse des femmes est de 3,3 (é.-t $=2,8$ ); c'est 0,4 point de plus que le niveau moyen de détresse des hommes, dont la cote autorapportée se situe à 2,9 (é.-t = 2,4). Les résultats du t-test indiquent que cette différence est significative au seuil de $1 \%: t=-1,8 ; \mathrm{p}=0,067$.
En moyenne, le niveau de détresse des patientes atteintes de cancer du sein est de 3,3 (é.-t $=2,8$ ), soit 0,1 point de plus que le niveau moyen de détresse des patients atteints de cancers hématologiques, une différence non significative $(\mathrm{t}=-0,614 ; \mathrm{p}=0,539)$.

Notons que la moyenne des personnes atteintes de cancer hématologique baisse à 2,6 au temps 2 (une diminution de 0,6$)$. Celle des patientes atteintes de cancer du sein baisse également, mais plus légèrement : la moyenne au temps 2 se situe à 3,1 (une diminution de 0,2). Cette différence n'est pas significative au temps $2(\mathrm{t}=-0,962 ; \mathrm{p}=0,338)$.

Les tableaux suivants présentent la cote au thermomètre selon le genre et le type de cancer au T1.

\section{Seuil clinique de référence au TD}

Au total, près de $34 \%(33,9 \%)$ des patients ont un score supérieur ou égal à 5 au thermomètre de la détresse. Il n'y a pas de différence significative selon le genre ni le type de cancer. Au temps 2, la proportion de patients ayant un score supérieur ou égal à 5 baisse à $28,1 \%$. On observe cependant une différence selon le genre au temps 2 : $33 \%$ des femmes atteignent le score clinique, contre 17,8 \% des hommes $\left(\mathrm{khi}^{2}=3,4 ; \mathrm{p}=0,06\right)$.

\section{Seuil clinique de référence anxiété et dépression}

Premièrement, concernant l'anxiété, un peu plus d'un patient sur $4(26,6 \%)$ présente un score supérieur ou égal à 5 au temps 1. L'anxiété est plus marquée chez les participantes que chez les participants. Ainsi au temps 1, 30,3\% des femmes atteignent le score clinique d'anxiété $\left(\mathrm{khi}^{2}=9,2 ; \mathrm{p}=0,002\right)$ contre $17,3 \%$ pour les hommes. Ces proportions diminuent au temps 2, mais la différence entre hommes et femmes reste significative : 9,8 \% des hommes contre 22,9\% des femmes $(\mathrm{p}=0,05)$.

Des différences significatives apparaissent aussi au temps 1 et 2 selon le type de cancer. Ainsi, au temps 1 , près de 1 patient sur 5 (19,8\%) atteint de cancer hématologique rencontre le seuil clinique, tandis que le tiers $(32,4 \%)$ des patientes atteintes de cancer du sein l'atteignent $\left(\mathrm{khi}^{2}=10,9 ; \mathrm{p}=0,001\right)$. Au temps 2, la différence persiste : 13,4\% des patients en hématologie pour $24,6 \%$ des patientes atteintes de cancer du sein ont un score de 5 ou plus $\left(\mathrm{khi}^{2}=3,03 ; \mathrm{p}=0,08\right)$.

Enfin, concernant la dépression, on constate que seuls 9,4 \% des patients ont un score supérieur ou égal à 5 au temps 1. Au temps 2, la proportion de patients ayant un score supérieur ou égal à 5 reste stable, puisqu'ils représentent 9,5\% des patients. Cependant, on observe une différence significative selon le genre au temps 1 : 5,4\% des hommes contre $11 \%$ des femmes atteignent le score clinique pour la dépression au temps $1\left(\mathrm{khi}^{2}=3,8 ; \mathrm{p}=0,05\right)$. Bien que les proportions restent identiques au temps 2 (5,8 \% des hommes contre 11,5\% des femmes), cette différence n'est plus significative. Par ailleurs, 7,2 \% des patients atteints de cancer hématologique ont un score de 5 ou plus, tandis qu'elles sont 11,4\% des patientes atteintes de cancer du sein à atteindre ce seuil. On observe les mêmes proportions pour le second temps de mesure. Aucune différence n'est significative selon le type de cancer, ni au temps 1 ni au temps 2 pour ce qui est de la dépression. 


\section{Évolution dans le temps des scores cliniques}

$T D$

Les résultats des analyses qui suivent sont issus d'ANOVA à mesures répétées. Ces résultats ont permis d'identifier une baisse significative de la cote au thermomètre entre le temps 1 et le temps 2 , et ce, pour l'ensemble de l'échantillon [F $(1 ; 1)=3,62 ; \mathrm{p}=0,06]$.

Cependant, les résultats des analyses n'ont pas montré d'effet significatif du terme d'interaction temps*type de cancer $[\mathrm{F}(1 ; 1)=0,07 ; \mathrm{p}=0,787]$. Ainsi, lorsque l'on s'intéresse à l'évolution selon le type de cancer, on observe une baisse significative de la cote au thermomètre entre le temps 1 et le temps 2 , quel que soit le type de cancer. Les effets de l'aide sur la cote au thermomètre a été comparée pour les deux groupes de patients (aide acceptée versus non acceptée) dans le temps, la variable d'intérêt étant la cote au thermomètre. Les résultats des analyses ne montrent pas d'effets significatifs de l'aide sur la diminution de la cote au thermomètre $(F=1,05 ; \mathrm{p}=0,3)$. Cependant, ils montrent que les deux groupes ont des cotes moins élevées au temps 2 qu'au temps 1 ( $F=4,3 ; p=0,04)$. Le niveau de détresse moyen baisse donc significativement entre le temps 1 et le temps 2, que les patients aient accepté l'aide ou non.

\section{Scores cliniques anxiété et dépression}

Nous observons une baisse du nombre de patients présentant un score clinique de l'anxiété dans le temps. Les analyses montrent que cette différence de proportion est significative pour les femmes entre le temps 1 et le temps $2\left(\mathrm{khi}^{2}=19,20\right.$; $\mathrm{p}=0,000$ ). Ainsi, par exemple, moins de femmes atteignent le score clinique d'anxiété au temps 2 ( $\mathrm{N}=20)$ qu'au temps 1 $(\mathrm{N}=27)$. Toutefois, les résultats des ANOVA à mesures répétées montrent que le score moyen d'anxiété diminue dans le temps aussi bien pour les patientes ayant accepté l'aide que pour ceux l'ayant refusée, au seuil de $1 \%$. ( $F=2,9 ; p=0,09)$.

Concernant le score clinique de dépression, les résultats des tests de $\mathrm{khi}^{2}$ montrent une différence significative de proportion entre le temps 1 et 2 . Ainsi, on constate que moins de patients présentent un score clinique de dépression au temps 2 qu'au temps 1, aussi bien chez les hommes $\left(\mathrm{khi}^{2}=4,117\right.$; $\mathrm{p}=0,042)$ que les femmes $\left(\mathrm{khi}^{2}=23,602 ; \mathrm{p}=0,000\right)$ et selon le type de cancer. Cependant, les résultats des ANOVA à mesures répétées montrent que les scores moyens de dépression n'évoluent pas dans le temps $(F=0,2 ; p=0,6)$. On observe donc une stabilité de l'intensité du score de dépression, que les patients aient accepté l'aide ou non.

\section{Problèmes présentés pendant la dernière semaine}

Les patients se sont exprimés sur les problèmes rencontrés lors de leur parcours. Une liste de 26 problèmes/préoccupations leur a été présentée, regroupée en six catégories (problèmes pratiques, sociaux, émotionnels, spirituels, informationnels et physiques). En moyenne, au temps 1, les patients déclarent 4,9 problèmes (é.-t. $=4$ ) et 3,7 problèmes au temps 2 (é.-t. = 3,2). Le graphique ci-dessous présente le pourcentage de patients ayant coché les différents nombres de problèmes.

Par ailleurs, c'est dans la catégorie problèmes émotionnels que les pourcentages de problèmes sont les plus élevés. Ainsi, seul le quart des patients $(25,4 \%)$ n'éprouve aucun problème dans cette catégorie alors qu'ils sont plus du trois quarts $(77,8 \%)$ à n'avoir coché aucun problème dans la sphère spirituelle. Le tableau 3 présente le nombre d'items cochés par catégories de problèmes.

Les graphiques suivants présentent les divers résultats pour chaque catégorie de problèmes au temps 1 et 2 pour l'ensemble de l'échantillon.

Graphique 1. Nombre de problèmes aux temps 1 et 2

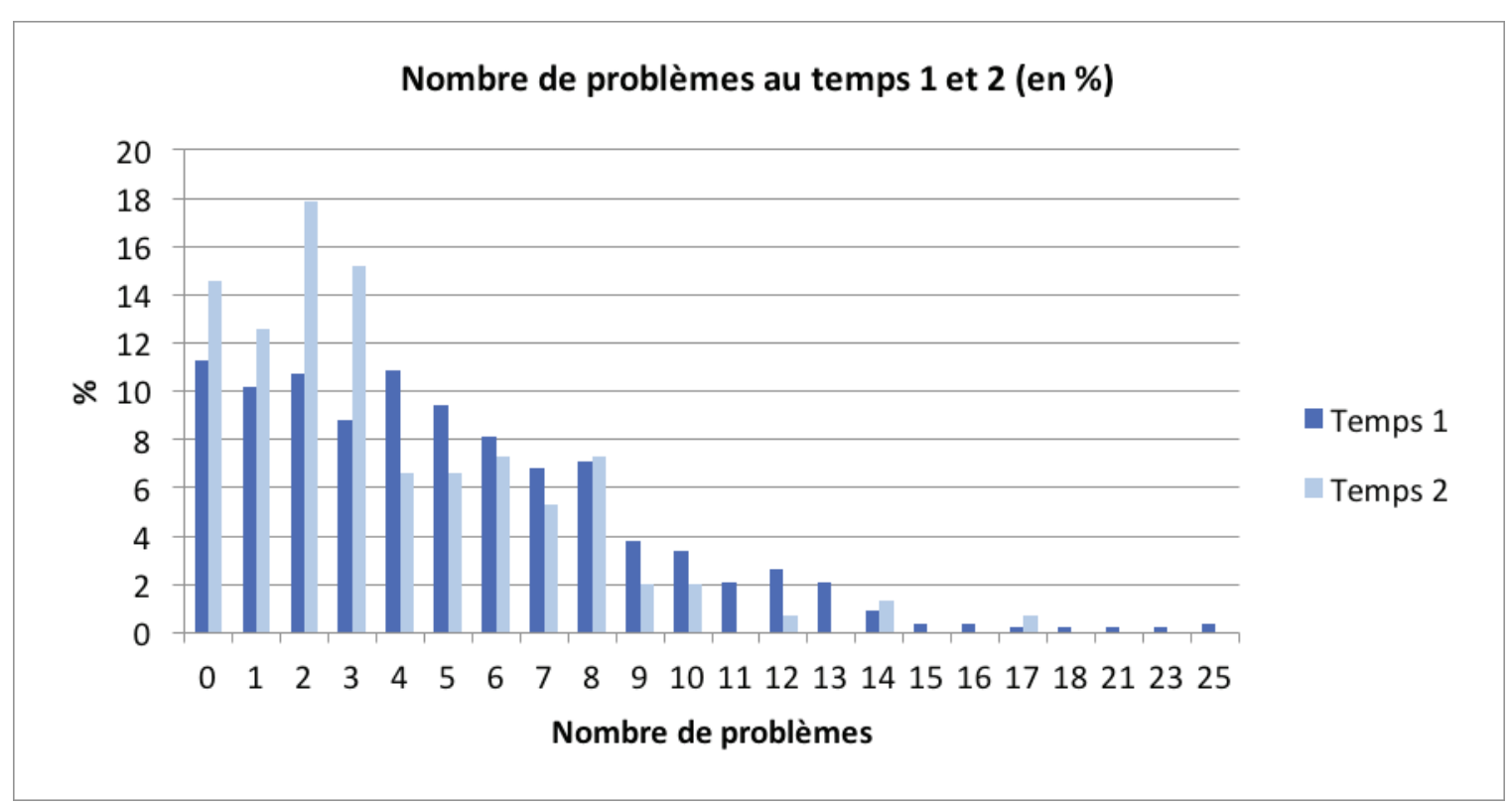


Tableau 3 : Nombre d'items sélectionnés par catégories de problèmes au temps 1

\begin{tabular}{|l|l|l|l|l|l|l|l|l|l|}
\hline & \multicolumn{7}{|l|}{ Nombre d'items par catégorie de problèmes (en \%) } \\
\hline Catégories de problèmes & $\begin{array}{l}\text { Nbre d'items } \\
\text { total }\end{array}$ & $\mathbf{0}$ item & $\mathbf{1}$ item & $\mathbf{2}$ items & $\mathbf{3}$ items & $\mathbf{4}$ items & $\mathbf{5}$ items & $\mathbf{6}$ items & $\mathbf{7}$ items \\
\hline PB Pratiques & 4 & 64,2 & 24,1 & 9,8 & 1,1 & 0,8 & & \\
\hline PB Sociaux & 4 & 48,6 & 32,4 & 14,7 & 3,6 & 0,8 & & \\
\hline PB Émotionnels & 7 & 25,4 & 29 & 19,4 & 14,1 & 6,6 & 3,4 & 0,8 & 1,3 \\
\hline PB Spirituels & 3 & 77,8 & 18,1 & 3 & 1,1 & & & \\
\hline PB Informationnels & 4 & 57 & 23,4 & 11,3 & 4,2 & 4,2 & & & \\
\hline PB Physiques & 4 & 35,7 & 33,5 & 21,6 & 7 & 2,3 & & \\
\hline
\end{tabular}

Graphique 2. Problèmes pratiques rencontrés

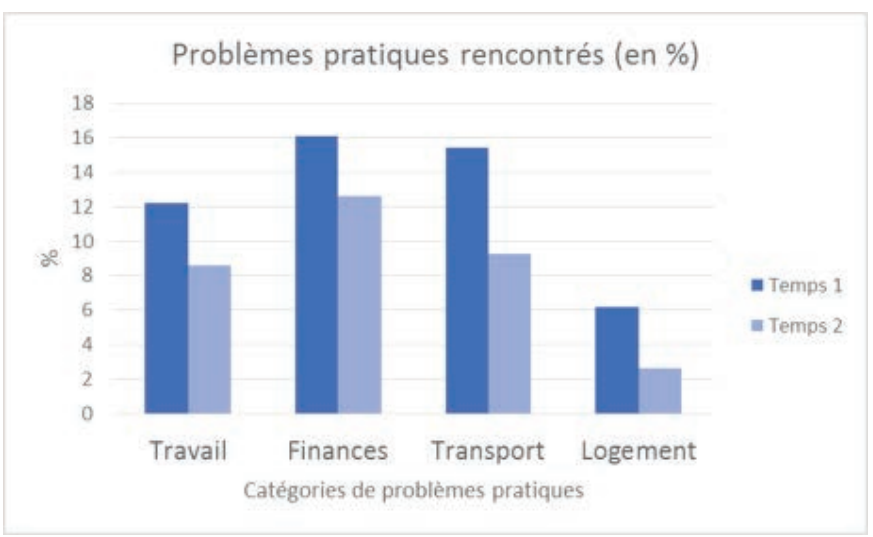

Graphique 4. Problèmes émotionnels rencontrés

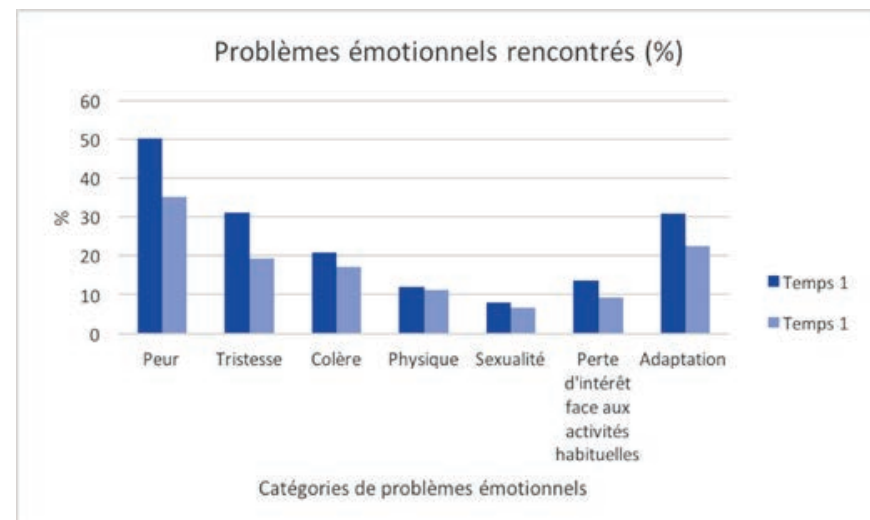

Graphique 6. Problèmes informationnels rencontrés

Problèmes relatifs à l'information rencontrés (\%)

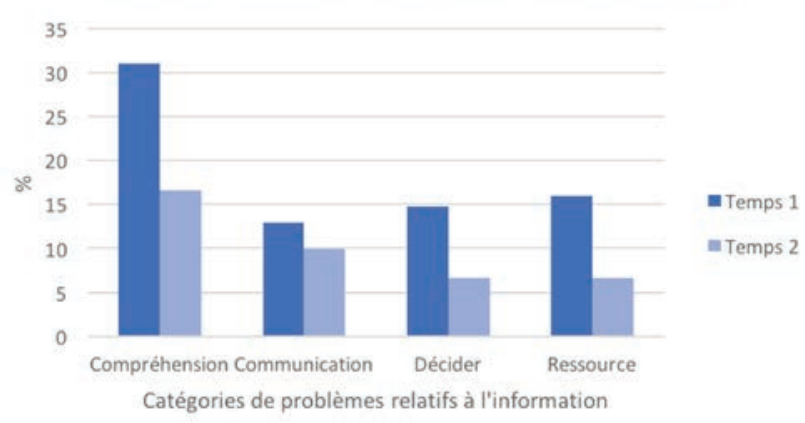

Graphique 3. Problèmes sociaux rencontrés

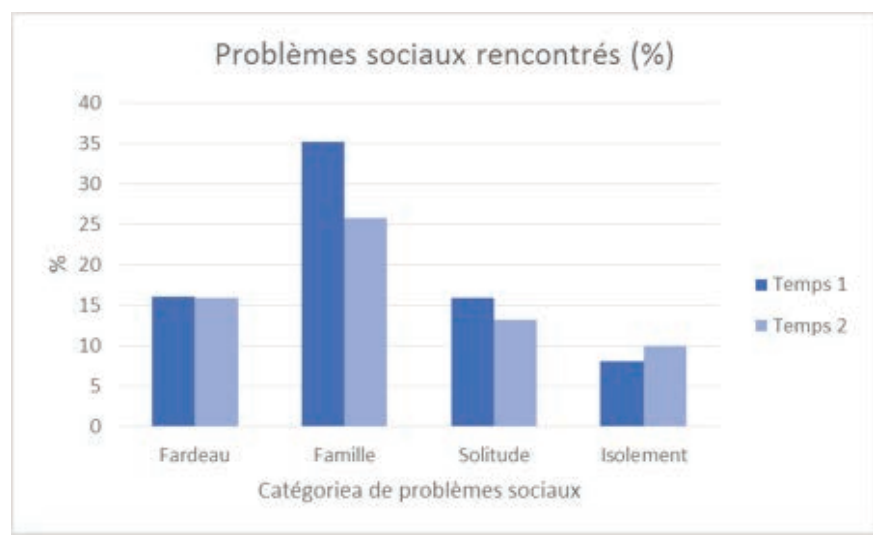

Graphique 5. Problèmes spirituels rencontrés

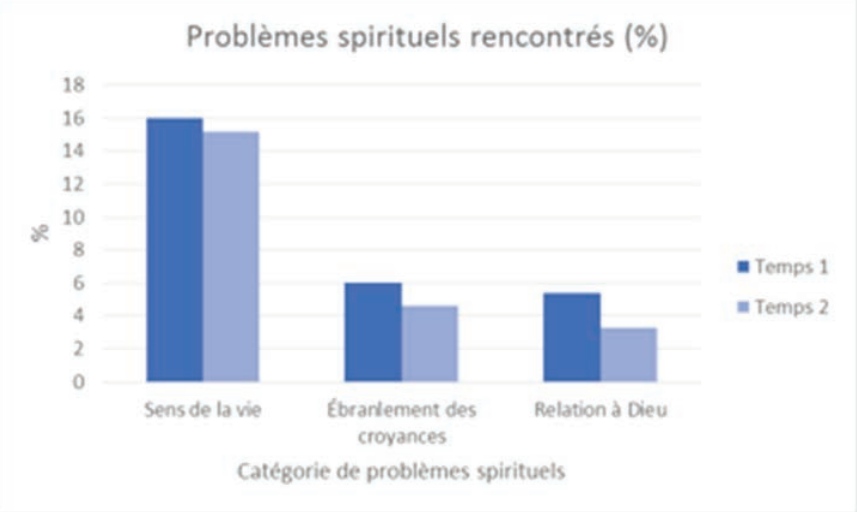

Graphique 7. Problèmes physiques rencontrés

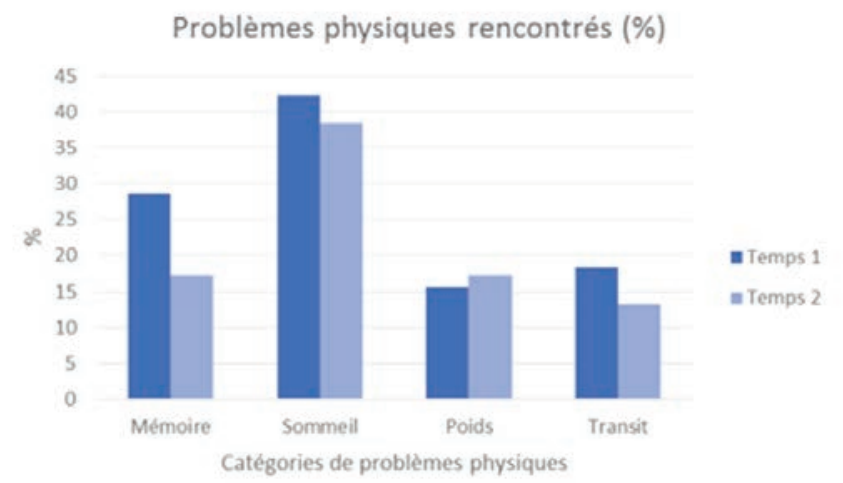


Graphique 8. Scores moyens des différents symptômes au temps 1 et 2.

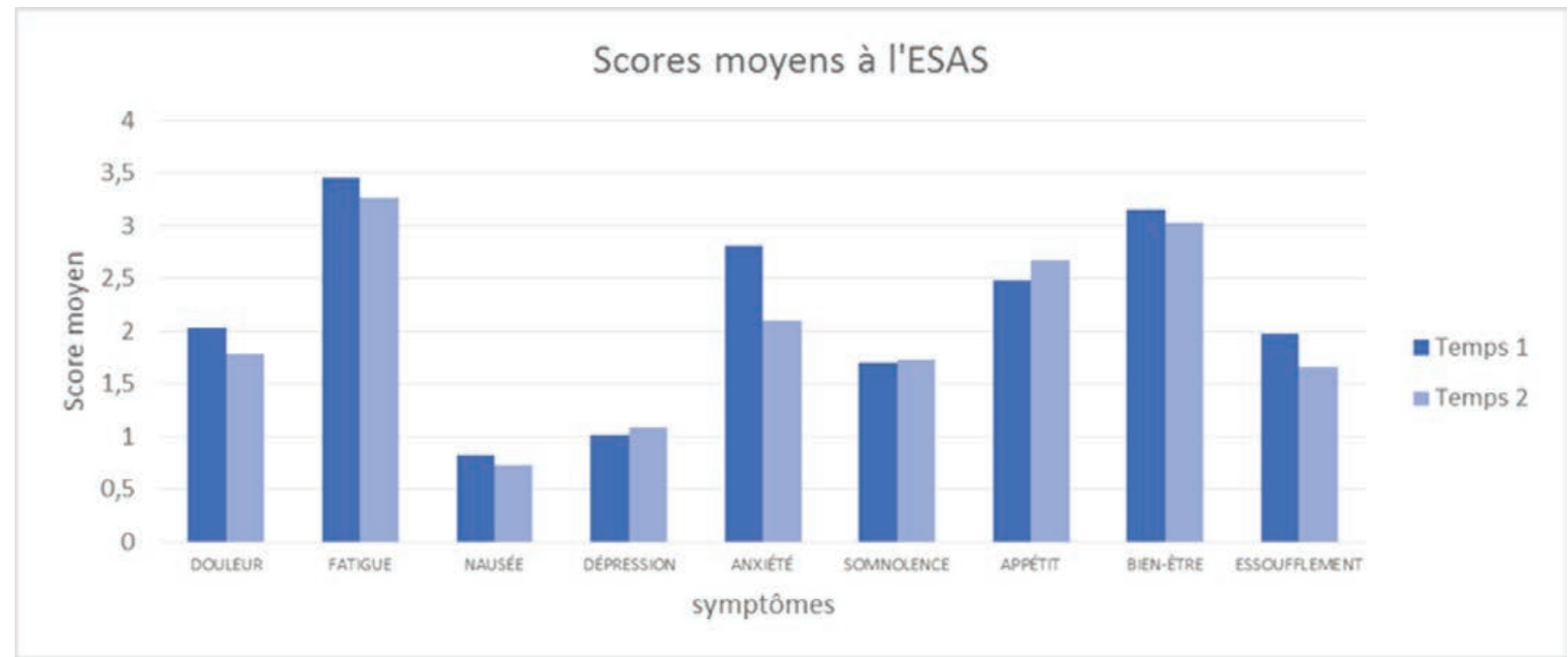

\section{Synthèse des problèmes rencontrés}

Dans l'ensemble, les problèmes les plus fréquemment rencontrés concernent, par ordre décroissant : la peur $(50 \%)$, le sommeil (42\%), la famille (35\%), la compréhension de la maladie et du traitement (31\%), l'adaptation face à la maladie $(31 \%)$ et la tristesse $(31 \%)$. Ainsi, les problèmes émotionnels semblent être un des sujets de préoccupation majeure pour les patients atteints de cancer, tandis qu'ils sont peu nombreux à déclarer des problèmes d'ordre spirituel.

Hommes et femmes ne sont toutefois pas égaux face aux problèmes rencontrés. Lorsque des différences significatives apparaissent via des tests de $\mathrm{khi}^{2}$, ce sont les femmes qui déclarent rencontrer le plus de problèmes. Au temps 1 , il y a 15 différences significatives selon le genre (sur 26 variables). Sur ces 15 différences significatives, les femmes sont plus nombreuses que les hommes à avoir signalé vivre un problème pour 11 variables. Ainsi les femmes rapportent plus fréquemment que les hommes avoir peur $(53 \%$ des femmes versus $44 \%$ des hommes; $\mathrm{khi}^{2}=3,2 ; \mathrm{p}=0,07$ ), ressentir de la tristesse $\left(34 \%\right.$ des femmes versus $24 \%$ des hommes; $\mathrm{khi}^{2}=4,3$; $p=0,037)$, rencontrer des changements d'apparence $(14 \%$ des femmes versus $6 \%$ des hommes; $\mathrm{khi}^{2}=5,78 ; \mathrm{p}=0,016$ ) et une perte d'intérêt face aux activités habituelles $(15 \%$ des femmes versus $9 \%$ des hommes; $\mathrm{khi}^{2}=3,49 ; \mathrm{p}=0,062$ ), devoir s'adapter à la maladie (34\% des femmes versus $22 \%$ des hommes; $\left.\mathrm{khi}^{2}=6,74 ; \mathrm{p}=0,009\right)$, se questionner dans leur relation avec Dieu (5\% des femmes versus $0 \%$ des hommes; $\left.\mathrm{khi}^{2}=2,72 ; \mathrm{p}=0,09\right)$, être préoccupés par la compréhension du traitement et de la maladie ( $34 \%$ des femmes versus $22,5 \%$ des hommes; $\left.\mathrm{khi}^{2}=7,1 ; \mathrm{p}=0,08\right)$, la prise de décision ( $17 \%$ des femmes versus $9 \%$ des hommes; $\mathrm{khi}^{2}=2,9 ; \mathrm{p}=0,08$ ), l'accessibilité des ressources (18\% des femmes versus $9 \%$ des hommes; $\left.\mathrm{khi}^{2}=5,6 ; \mathrm{p}=0,02\right)$, rencontrer des problèmes de mémoire $(32 \%$ des femmes versus $20 \%$ des hommes; $\left.\mathrm{khi}^{2}=6,8 ; \mathrm{p}=0,009\right)$ et de sommeil $(47 \%$ des femmes versus $36 \%$ des hommes; $\mathrm{khi}^{2}=9,1 ; \mathrm{p}=0,01$ ).
Par ailleurs, hommes et femmes ne font pas face aux mêmes difficultés : les hommes sont plus touchés par des problèmes de sexualité $(11,9 \%$ des hommes versus $6 \%$ des femmes; $\left.\mathrm{khi}^{2}=4,21 ; \mathrm{p}=0,04\right)$, d'isolement neutropénique $(12,6 \% \mathrm{vs}$ $6 \%$; $\left.\mathrm{khi}^{2}=5,79 ; \mathrm{p}=0,02\right)$, de travail $\left(16,6 \%\right.$ vs $10 \% ; \mathrm{khi}^{2}=3,74$; $\mathrm{p}=0,05)$ et de finances $\left(20,5 \%\right.$ vs $14 \%$; $\left.\mathrm{khi}^{2}=3,01 ; \mathrm{p}=0,08\right)$. Les femmes quant à elles rapportent plus fréquemment de problèmes émotionnels et de problèmes relatifs à l'information.

$\mathrm{Au}$ temps 1, on constate 10 différences significatives selon le type de cancer. Sur ces 10 différences, 4 touchent particulièrement les patients atteints de cancer hématologique : sexualité $(11,5 \%$ versus $5 \%$ pour les patientes atteintes de cancer du sein; $\left.\mathrm{khi}^{2}=7,19 ; \mathrm{p}=0,007\right)$, isolement de protection $\left(13,2 \%\right.$ vs $3 \%$; $\left.\mathrm{khi}^{2}=15,6 ; \mathrm{p}=0,000\right)$, finances $(21,0 \%$ vs $12 \%$; $\left.\mathrm{khi}^{2}=7,7 ; \mathrm{p}=0,005\right)$, se sentir un fardeau $(20,2 \%$ vs $13 \% ; \mathrm{khi}^{2}=5,36 ; \mathrm{p}=0,02$ ), soit à peu de chose près les mêmes différences observées selon le genre. À l'inverse, les patientes atteintes de cancer de sein sont plus nombreuses que les patients atteints de cancer hématologique à déclarer avoir peur (54\% des cancers du sein versus $45 \%$ des cancers hématologiques; $\mathrm{khi}^{2}=3,98 ; \mathrm{p}=0,05$ ), ne pas avoir d'intérêt pour les activités habituelles ( $17 \%$ vs $9 \%$; $\mathrm{khi}^{2}=5,5 ; \mathrm{p}=0,019$ ), être préoccupés par la compréhension du traitement et de la maladie ( $34 \%$ vs $27 \%$; $\left.\mathrm{khi}^{2}=3,1 ; \mathrm{p} .=0,08\right)$, la prise de décision ( $17 \%$ vs $\left.12 \% ; \mathrm{khi}^{2}=2,9 ; \mathrm{p}=0,08\right)$, être préoccupés par les ressources $\left(19 \%\right.$ vs $12 \%$; $\left.\mathrm{khi}^{2}=6,5 ; \mathrm{p}=0,01\right)$ et rencontrer des problèmes de sommeil ( $47 \%$ vs $36 \%$; $\mathrm{khi}^{2}=7,1 ; \mathrm{p}=0,03$ ).

D'un point de vue temporel, on constate qu'il existe moins de différence significative au temps 2 qu'au temps 1 . Les problèmes émotionnels semblent néanmoins persister dans le temps, puisque l'on observe encore 6 différences selon le genre au temps 2 .

\section{Symptômes présentés}

Au temps 1, comme au temps 2, la médiane du nombre de symptômes se situe à 5 . La valeur modale au temps 1 se situe à 6 symptômes, avec un pourcentage de 15,6\%. Au temps 2, 
il y a deux modes : 4 et 5 symptômes, avec un pourcentage de $17,2 \%$ pour ces deux valeurs. Hommes et femmes présentent un nombre similaire de symptômes, avec une moyenne de 2,4 symptômes par patients. Au temps 1 et 2, il n'y a pas de différence significative selon le genre $\left(\mathrm{khi}^{2}=14,3 ; \mathrm{p}=0,11\right)$ ni selon le type de cancer $\left(\mathrm{khi}^{2}=9,8 ; \mathrm{p}=0,37\right)$.

Le graphique 8 présente les scores moyens des différents symptômes au temps 1 et 2 .

Les trois symptômes pour lesquels l'intensité est la plus élevée au temps 1 sont : la fatigue (avec une moyenne de 3,46), le bien-être (moyenne de 3,16), et l'anxiété (moyenne de 2,81). Fatigue et bien-être demeurent presque aussi présents au temps 2 (moyennes respectives de 3,26 et de 3,03), l'appétit vient au troisième rang (moyenne est de 2,67). Ainsi, on observe une relative stabilité de l'intensité des symptômes entre le temps 1 et 2 , les symptômes les moins forts et les plus forts étant les mêmes aux temps 1 et 2 . On observe également une diminution de l'intensité pour presque tous les symptômes, à l'exception de la dépression, de la somnolence et de l'appétit.

\section{Explication de la variation}

Le genre et le type de cancer sont intimement liés dans cette étude. Afin de déterminer si les différences observées sont attribuables au genre uniquement, au type de cancer uniquement ou au deux, des régressions linéaires multiples ont été utilisées. Il est intéressant de noter que les variables indépendantes (genre et type de cancer) n'ont pas toutes un effet significatif. Nos résultats montrent que seule la variable genre à un impact significatif $(\mathrm{B}=0,7 ; \mathrm{p}=0,05)$ sur la cote $a u$ thermomètre, tandis que le type de cancer n'a pas d'effets significatifs $(B=-0,2 ; p=0,39)$. Concernant les symptômes, les résultats montrent que le type de cancer a un effet significatif sur l'appétit $(B=-0,64 ; p=0,059)$ et la douleur $(B=-0,57$; $\mathrm{p}=0,059)$. Ainsi pour ces deux variables, les patients atteints de cancers hématologiques ont des scores plus élevés. Par contre, il n'y a pas d'effet de genre.

Le genre a pour sa part un effet significatif sur la fatigue $(B=0,67 ; p=0,06)$, la dépression $(B=0,39 ; p=0,03)$, l'anxiété $(\mathrm{B}=0,64 ; \mathrm{p}=0,01)$. À ce propos, le fait d'être une femme est associé à une augmentation de l'intensité des symptômes sur ces variables. Il n'y a pas d'effet significatif du type de cancer sur ces variables.

Il est rare que les deux variables jouent un rôle de manière simultanée, hormis le cas exceptionnel des nausées où il y a un effet du genre $(B=0,53 ; p=0,04)$ et du type de cancer $(B=-0,47 ; p=0,04)$. Ces deux facteurs étant cumulatifs, ce sont les femmes atteintes de cancers hématologiques qui sont le plus durement frappées.

On constate que le type de cancer ou le genre ont un effet significatif sur les variables d'intérêt, mais rarement les deux en même temps. Lorsque le genre a un effet significatif, c'est au détriment des femmes, qui ont alors des scores plus élevés que les hommes. Lorsque c'est le type de cancer qui a un effet significatif, ce sont les patients atteints de cancer hématologique qui ont des scores plus élevés, quel que soit le genre du patient.
Intéressons-nous maintenant aux effets du nombre de symptômes et de problèmes sur la cote au thermomètre. La valeur des coefficients non standardisés indique dans quelle mesure la cote au thermomètre varie en fonction d'une variable indépendante. Ainsi, nous constatons que pour chaque problème supplémentaire rapporté par le patient, sa cote au thermomètre va augmenter de 0,28 au temps 1 , et de 0,46 au temps 2. Donc, un patient qui rapporte 10 problèmes au temps 1 aura une cote de 2,8 points de plus qu'un patient qui n'en rapporte aucun (4,6 points de plus pour le temps 2$)$. Nous constatons également aux regards des résultats de ces analyses que le nombre de problèmes influence davantage au temps 2 comparativement au temps 1 . Pour les deux temps de mesure, l'effet du nombre de problèmes est plus fort que l'effet du nombre de symptômes.

\section{Aide souhaitée}

À la question « Souhaitez-vous avoir de l'aide pour l'un des problèmes nommés ci-haut? » :

- $22,7 \%$ souhaitent recevoir une aide au temps 1

- $14,7 \%$ souhaitent recevoir une aide au temps 2

Aucune différence significative reliée au genre ou au type de cancer, que ce soit au temps 1 ou temps 2, n’a été constatée.

\section{Références offertes et acceptées}

Au temps de mesure 1, les références les plus offertes aux patients sont, par ordre décroissant, l'oncopsychologue $(26,4 \%)$, le travailleur social $(4,1 \%)$, la conseillère clinicienne en soins infirmiers (3,5\%). À l'inverse, les références les moins proposées aux patients sont : le physiothérapeute $(0,2 \%)$, le pharmacien $(0,2 \%)$, un autre établissement $(0,2 \%)$, une infirmière $(1,1 \%)$.

Dans la majorité des cas, seule une référence est proposée (88\%). Les femmes sont plus nombreuses que les hommes à être orientées vers un oncopsychologue (27,4\% contre 23,6\%) ou vers un travailleur social (5,1\% contre 1,6\%). À l'inverse, les hommes sont plus orientés vers un médecin (4,7\% contre $1,5 \%)$ ou un nutritionniste (5,5\% contre 1,8\%).

Pareillement, les patientes atteintes du cancer du sein sont plus nombreuses que les patients atteints de cancer hématologique à être orientées vers un oncopsychologue $(29,2 \%$ contre $22,8 \%$ ou vers un travailleur social (5,9\% contre $1,9 \%)$. À l'inverse, les patients atteints de cancer hématologique sont plus orientés vers un médecin $(2,9 \%$ contre $2 \%$ ) ou un nutritionniste $(5,3 \%$ contre $0,8 \%)$.

La référence est acceptée par le patient dans $40 \%$ dans cas au temps 1, et $33 \%$ des cas au temps 2 . Il n'y a pas de différence significative selon le genre au temps 1, c'est-à-dire que les hommes et les femmes acceptent la référence dans des proportions similaires. Par contre, au temps 2, on observe que ce sont les femmes qui acceptent plus fréquemment l'aide que les hommes. Elles sont $42 \%$ à l'accepter, contre $16 \%$ des hommes $\left(\mathrm{khi}^{2}=6,45 ; \mathrm{p}=0,01\right)$. Concernant le type de cancer, il n'y a pas de différence significative au temps 1 . La différence est cependant significative au temps $2: 23 \%$ des patients atteints de cancers hématologiques acceptent la référence, contre $50 \%$ des patientes atteintes de cancer du sein $\left(\mathrm{khi}^{2}=7,03 ; \mathrm{p}=0,08\right)$. 
Les références sont plus fréquemment acceptées par les patients à certains moments particuliers de leur trajectoire. Les patients en hémato-oncologie acceptent plus fréquemment l'aide pendant le traitement : 62,5 \% d'acceptation $\left(\mathrm{khi}^{2}=4,8\right.$; $\mathrm{p}=0,02)$. Les patients en hémato-oncologie acceptent également plus l'aide au moment de l'admission à l'unité : $45 \%$ d'acceptation. À l'inverse, elle est plus fréquemment refusée par les patients au moment de la consultation ou du début du traitement en hémato-oncologie : $81 \%$ de refus $\left(\mathrm{khi}^{2}=4,1\right.$; $\mathrm{p}=0,042$ ), au moment de la fin du traitement en hémato-oncologie : 85,7\% de refus $\left(\mathrm{khi}^{2}=3,5 ; \mathrm{p}=0,06\right)$.

\section{Limites de la phase 1 de l'étude}

Malgré les efforts consentis au suivi pour l'implantation de l'outil de dépistage de la détresse auprès des équipes (comité clinique et scientifique, focus group, formation, rencontre d'échanges) et par le fait même, la collecte de données, plusieurs données étaient manquantes dans les suivis de l'outil de dépistage. Par ailleurs, avec le recul, il convenait moins à une clientèle traitée pour un cancer hématologique. Nous avons donc choisi ici de ne pas présenter certains résultats se rapportant à cet outil. Nos résultats sont cependant très robustes en ce qui concerne les portraits de la détresse présentée par notre clientèle visée par l'étude à deux temps différents de la trajectoire de soins et nous sommes d'avis qu'ils peuvent être généralisés, même si certains aspects en lien avec les ressources seraient sans doute différents.

\section{DISCUSSION}

Notre étude confirme certains résultats d'études antérieures, notamment en ce qui concerne la prévalence de la détresse. En effet, près de $34 \%$ de notre échantillon rencontre le seuil clinique de détresse au temps 1, ce résultat est similaire aux résultats rapportés en introduction. Par ailleurs, plus du quart des patients $(26,6 \%)$ atteignent le seuil clinique d'anxiété et autour de 9,4 le seuil clinique de référence pour la dépression (ces seuils, rappelons-le, nécessitent une évaluation en psycho-oncologie). La prévalence de l'anxiété rapportée dans notre étude est plus élevée que celle décrite par Mitchell et collègues (2013) dans une vaste méta-analyse comparant des survivants du cancer à des personnes en bonne santé. Seize études ont évalué la dépression et 10 ont évalué l'anxiété. Il en ressort une prévalence de l'anxiété de l'ordre de près de $18 \%$ (17,9\%) chez les survivants de cancer ( $\mathrm{N}=48$ 964) comparativement à un taux de $13,9 \%$ chez une population non atteinte $(\mathrm{N}=226$ 467). Le taux de dépression était de 11,6 \% ( $\mathrm{N}=51381)$ chez les survivants du cancer et de 10,2 \% dans le groupe contrôle ( $\mathrm{N}=217$ 630). Comme cette vaste étude porte chez des personnes diagnostiquées de cancer depuis au moins deux ans et que l'échantillon de notre étude comporte quant à elle une majorité de femmes nouvellement diagnostiquées de cancer du sein (moins de 3 semaines) et qu'un certain nombre de patients atteints de cancers hématologiques venaient d'être diagnostiqués (quoique ce nombre réel soit inconnu) ceci pourrait en partie expliquer la différence observée en ce qui concerne l'anxiété. En effet, on sait que de hauts niveaux d'anxiété sont communs chez les personnes nouvellement diagnostiquées et ces niveaux baissent avec l'ajustement à cette nouvelle réalité (Hammeleft, 2015).

De plus, une autre étude de Mitchell réalisée en 2007 a comparé l'efficacité d'outils brefs de dépistage tels que le thermomètre de la détresse versus l'utilisation d'une échelle standardisée comme le HADS (Hospital Anxiety and Depression Scale), le BDI (le Beck Depression Inventory) et les entrevues cliniques. Mitchell dans cette étude indique que les outils de dépistage courts tels que ceux utilisés dans le cadre de notre étude sont plus efficaces à détecter la présence ou non du symptôme qu'à indiquer un trouble cliniquement significatif (Mitchell, 2007). Ainsi selon Mitchell (2007) 11 patients sur 20 qui atteignent le seuil de référence pour l'anxiété sont cliniquement anxieux, alors que ce sont 7 patients sur 20 qui atteignent le seuil de référence qui sont cliniquement dépressifs. Par contre, sur 20 patients testés négatifs, 1 seul cas de dépression a été manqué alors que dans le cas de l'anxiété, ce sont 4 cas qui ont été manqués. Par ailleurs, pour la détresse, le thermomètre génère 9 faux positifs et 4 faux négatifs. Comme le souligne Mitchell, le seuil de césure du thermomètre peut bien sûr être modifié, mais un gain en sensibilité augmenterait la perte en spécificité et vice versa. D'ailleurs, comme l'indique Coyne (2013), bien que les outils courts de dépistage aient été validés, il n'en est pas de même pour les Cut-off, seuils de césure de ces outils. C'est pour ces raisons que l'on s'accorde à dire que le dépistage de la détresse, à l'instar de tout test de dépistage, est le début ou la poursuite d'une discussion et d'une évaluation, et non une finalité en soi.

Les résultats de notre étude ont généré plusieurs questions. Par exemple, dépister la détresse permet-il d'améliorer le devenir des personnes atteintes de cancer? Il s'agit en fait d'une double question. La première étant : les interventions elles-mêmes sont-elles efficaces pour réduire la détresse? Et en deuxième, le dépistage permet-il d'améliorer les résultats de soins? Il existe aujourd'hui peu de littérature démontrant l'efficacité des interventions psychosociales et psychopharmacologiques sur la réduction de la détresse (Coyne, 2013). La deuxième question demeure quant à elle entière, comme l'affirment également Faller, Schuller, Richard, Heckel, Weis et Kuffner (2013) et Salmon, Clark, McGrath et Fisher (2014). D'une part, notre étude montre bien que le niveau de détresse diminue dans le temps pour notre échantillon. Cependant, cette baisse survient peu importe que l'aide ait été acceptée ou non, ce résultat allant dans le même sens que d'autres études (Henselmans, Helgeson, Seltman, de Vries, Sanderman et Ranchor, 2010; Lam, Bonnano, Mancini et al., 2010, cités par Coyne, 2013) à l'effet que la détresse peut être limitée dans le temps et se résoudre sans services spécialisés. À la lumière de ces résultats et de notre expérience clinique, nous sommes enclins à penser que les personnes atteintes de cancer ont de nombreuses ressources internes et familiales qui contribuent à leur adaptation et à leur résilience dans le temps, et qui peuvent être renforcées et honorées. Nous traiterons davantage de cet aspect dans un deuxième article.

D'autre part, il faut aussi réaliser ici que la détresse aurait pu tout aussi bien augmenter dans le temps, car le temps 2 correspond pour un grand nombre de patients de notre 
échantillon à des périodes intenses de traitement (femmes atteintes de cancer du sein en radiothérapie ou chimiothérapie). De plus, le temps 2 correspond pour d'autres au moment du congé (patients atteints de cancers hématologiques/greffés) et où majoritairement les effets de la thérapie se font encore sentir et la peur de la récidive ou de la progression peut survenir, celle-ci étant un des éléments les plus fréquemment associés à la détresse psychologique (Herschback, Keller, Knight et al., 2004, cités dans Suchocka-Capuono et Bungener, 2010). En plus, nous ne savons pas non plus avec certitude quels ont été les soins offerts et donnés outre la référence. Il est donc possible que ces autres interventions réalisées auprès de notre échantillon aient été utiles. De plus, nous savons que le suivi de l'outil de dépistage de la détresse a été dans bien des cas insuffisamment complétés de l'aveu des infirmières rencontrées en cours d'implantation à plusieurs reprises. Cette observation doit être considérée d'autant plus que les infirmières ne documentent pas nécessairement toutes leurs interventions de nature psychosociale, voire même les conseils donnés sur les différents symptômes. Cela ayant été constaté à maintes reprises par notre équipe de chercheurs, ce qui rend toute analyse fort périlleuse et nous incite à une grande prudence.

Notre étude a permis de dresser des portraits distincts de la détresse des deux populations ciblées, portraits présentant des airs de famille sur plusieurs plans, mais aussi avec ses particularités et ses défis. Notamment, elle a regroupé exceptionnellement une proportion importante d'hommes atteints de cancers hématologiques, ce qui nous a permis de mettre en évidence des effets de genre sur la détresse ainsi que quelques effets associés au type de cancer. De fait, nos résultats laissent entrevoir une possible influence des valeurs traditionnelles « masculines » sur les problèmes présentés et sur ce qui a trait à la demande d'aide. Ainsi, les hommes dans notre étude manifestent moins de détresse, en moyenne 0,4 point de moins que le niveau moyen de détresse des femmes, dont la cote autorapportée se situe à 3,3, et ce, malgré qu'ils fassent face en général à un pronostic beaucoup plus réservé que les femmes atteintes de cancer du sein. De plus, au temps 1, 17,3\% des hommes contre 30,3\% des femmes atteignent le score clinique d'anxiété. Ces proportions diminuent au temps 2, mais la différence entre hommes et femmes reste significative : $9,8 \%$ des hommes contre $22,9 \%$ des femmes. Bien qu'au temps 1 hommes et femmes soient similaires dans l'acceptation de l'aide, au temps 2, on observe que les femmes acceptent plus fréquemment l'aide que les hommes. Elles sont $42 \%$ à l'accepter, contre $16 \%$ des hommes. Ces résultats laissent croire qu'encore aujourd'hui, les hommes et les femmes sont vraisemblablement influencés pour se conformer aux stéréotypes de la féminité et de la masculinité. En ce sens, plusieurs auteurs ont mis en évidence que cette construction sociale de la masculinité influence la santé, la maladie ainsi que la façon dont les hommes vont exprimer leurs sentiments, minimiser leurs inquiétudes face à leur état de santé et préférer gérer leurs problèmes eux-mêmes (Berger, 2011; Galdas, Cheater et Marshall, 2005; Schofield, Connell, Walker, Wood et Butland, 2000). Selon Berger (2011), les valeurs accordées à l'autonomie, à l'estime de soi et à « l'égo masculin » expliqueraient en partie pourquoi les hommes demandent peu d'aide.

Toutefois, il faut aussi nuancer ces résultats. D’une part, comme mentionné plus tôt, davantage de femmes que d'hommes dans notre échantillon venaient tout juste d'être diagnostiquées. Il faut se questionner sur l'effet d'un récent diagnostic ou d'autres facteurs dans le niveau de détresse et l'acceptation de l'aide. D'autre part, il est aussi vrai que si l'homme atteint de cancer hématologique (de 10 ans plus jeune en moyenne que les femmes atteintes de cancer du sein et sur le marché du travail) rencontre davantage de difficultés associées au travail, aux finances et se perçoit davantage comme un fardeau, il est aussi isolé sur de longues périodes, immunosupprimé parfois pendant plusieurs mois, voire pendant des années après la greffe, et donc fréquemment incapable de reprendre le travail. Ainsi, les résultats obtenus pourraient témoigner tout autant de l'impact financier et social de certains traitements du cancer à certaines périodes de vie que d'une réalité socioculturellement construite.

Selon Fitch et collègues, les premiers enseignements que l'on tire des efforts concertés pour répondre aux besoins psychosociaux des personnes touchées par le cancer mettent l'emphase sur l'importance des infirmières en oncologie sur le plan du dépistage et des interventions précoces face à la détresse (Fitch et al., 2012). De réels obstacles à l'utilisation optimale de l'outil de dépistage ont surgi tout au long de l'étude. Cet aspect sera traité dans le prochain article. Mais il faut tout de même mentionner à ce sujet les écrits de Fulcher et GosselinAcomb (2007), qui indiquent que les infirmières sont préoccupées par la charge de travail et le manque de temps ainsi que par leurs habiletés à dépister la détresse (Arantzamendi et Kearney, 2004; Mitchell, Kaar, Coogan et Herdman, 2008 cités dans Fillion, Cook, Blais et al., 2011). Notre étude sur le terrain nous a permis de relever d'autres obstacles. En effet, il est à noter aussi que le manque d'intimité caractérisant les salles de traitement à aires ouvertes ne facilite pas la tenue de conversations d'ordre plus émotif entre professionnels et patients. Un autre aspect du dépistage de la détresse que nous devons considérer est l'inconfort de certains professionnels à questionner sur des sujets délicats. Plus il existe d'inconfort à parler d'un sujet, plus le temps requis pour le faire est grand, alors que lorsque le degré de confort augmente, plus les professionnels y consacrent du temps et moins cela prend de temps pour réaliser l'activité (communication personnelle avec Deborah McLeod, 2012. Journée de recherche sur le dépistage systématique de la détresse).

\section{Recommandations et conclusion}

À la lumière de ce que nous avons observé pendant l'étude, nous croyons nécessaire de faire ces recommandations d'ordre clinique et de recherche :

- Renforcer le volet de la formation des infirmières au dépistage, qui devrait inclure le renforcement des stratégies d'adaptation des personnes atteintes de cancer à travers un réel partenariat de soins.

- Encourager et soutenir les infirmières à mieux documenter leurs actions, particulièrement leurs interventions en psychoéducation ou de type soutien psychosocial. Selon une 
étude de Marinis, Piredda, Chiara Pascarella, Vincenzi, Spiga, Tartaglini, Alvaro et Matarase (2010), 40 \% seulement des actions de l'infirmière seraient documentées. Il faut donc que les éducateurs et les superviseurs cliniques des infirmières accordent une réelle importance à cet aspect.

- Accorder une attention plus soutenue à la demande de référence, particulièrement chez l'homme atteint de cancer. Il est nécessaire de développer des façons de faire pour mieux présenter les raisons et la pertinence de la référence, comment mieux accompagner le patient dans cette démarche et comment en suivre les résultats.

- Offrir un environnement et des conditions favorables pour la réalisation du dépistage de l'ODD.

- Intégrer les patients partenaires ressources dans le processus de l'ODD.

\section{RÉFÉRENCES}

Accréditation Canada (2010). Normes relatives au traitement du cancer. Consulté sur https://www.accreditation.ca/fr/traitement-du-cancer

Bathia, S., Francisco, L., Carter, A., Sun, C.-L., Baker, S., Gurney, J.G. et al. (2007). Late mortality after allogenic hematopoietic cell transplantation and functional status of long-term survivors; report from the BMT survivor study. Blood, 110(10), 3784-3792.

Berger, S. (2011). La signification de la demande d'aide pour des hommes atteints d'un cancer de la sphère oto-rhino-laryngologique (ORL). Mémoire présenté à la Faculté des études supérieures. Université de Montréal.

Bultz, B. et Carlson, L. (2006). Emotional distress: The sixth vital sign-future directions in cancer care. Psycho-Oncology, 15, 93-95. doi:10.1002/pon.102

Carlson, L.E., Angen, M., Cullum, J., Goodey, E., Koopmans, J., Lamont, L. et al. (2004). High levels of untreated distress and fatigue in cancer patients. British Journal of Cancer, 90, 2297-304. doi:10.1038/sj.bjc.6601887

Compas, B.E. et Luecken, L. (2002). Psychological Adjustment to Breast Cancer. Current directions in psychological sciences, 11(3), 111-114.

Coyne, J.C. (2013). Second thoughts about implementing routine screening of cancer patients for distress. Psycho-Oncology, 7, 243249. doi:10.1007/s11839-013-0437-z

Creswell, J.W. et Plano Clark, V.L. (2007). Designing and conducting mixed methods research. Thousand Oaks, CA: Sage Publications.

De Marinis, M.G. Piredda, M. Chiara Pascarella, M., Vincenzi, B., Spiga, F., Tartaglini, D., Alvaro, R. et Matarase, M. (2010). If it is not recorded, it has not been done!? Consistency between nursing records and observed nursing care in an Italian hospital. Journal of Clinical Nursing, 19, 1544-1552. doi: 10.1111/j.1365-2702.2009.03012.x

Faller, H., Schuler, M., Richard, M., Heckel, U., Weis, J. et Kuffner, R. (2013). Effects of psychooncologic interventions on emotional distress and quality of life in adult patients with cancer: Systematic review and meta-analysis. Journal of Clinical Oncology, 31(6), 782-793.

Fitch, M. (2008). Cadre des soins de soutien. Revue canadienne des soins infirmiers en oncologie, 18(1). doi:10.5737/1181912x1811524

Fitch, M., Howell, D., McLeod, D. et Green, E. (2012). Dépistage de la détresse; l'intervention est une fonction essentielle des infirmières en oncologie. Revue canadienne des soins infirmiers en oncologie, 22(1). doi:10.5737/1181912x2212130
- Poursuivre la recherche en explorant l'expérience de la personne atteinte de cancer à travers son expérience du dépistage afin de mieux saisir les opportunités de mieux la soutenir dans les différents contextes interactionnels.

Cette étude, en plus de dresser des portraits de détresse auprès de deux groupes distincts de patients atteints de cancer, a permis de soulever de nombreuses questions auxquelles nous avons tenté de trouver des réponses dans la phase 2 de l'étude, en comprenant mieux les perceptions des différents acteurs autour du dépistage systématique de la détresse. De plus, la réalisation de cette étude a offert aux chercheures, qui sont avant tout des cliniciennes, un terrain fertile aux discussions et réflexions pour l'implantation et la consolidation de la pratique infirmière à l'égard du dépistage.

Fillion, L., Cook, S., Blais, M.-C., Veillette, A.-M., Aubin, M., de Serres, M. et al. (2011). Implementation of screening for distress with professional cancer navigators. Oncologie (Dossier Après Cancer), 13, 277-289. doi:10.1007/s10269-011-2026-8

Foss, C. et Ellefsen, B. (2002). The value of combining qualitative and quantitative approaches in nursing research by means of method triangulation. Journal of Advanced Nursing, 40(2), 242-248.

Fulcher, C.D. et Gosselin-Acomb, T.K. (2007) Distress assessment: Practice change through guideline implementation. Clinical Journal of Oncology Nursing, 11, 817-821.

Galdas, P.M., Cheater, F. et Marshall, P. (2005). Men and health helpseeking behaviour: literature review. Journal of Advanced Nursing, 49, 616-623.

Groenvold, M., Petersen, M.A. Idler, E. Bjorner, J.B., Fayers, P.M. et Mouridsen, H.T. (2006). Psychological distress and fatigue predicted recurrence and survival in primary breast cancer patients. Breast Cancer Research and Treatment, 105, 209-219.

Grunfeld, E., Coyle, D., Whelan, T., Clinch, J., Reyno, L., Earle, C.C. et al. (2004). Family caregiver burden: Results of a longitudinal study of breast cancer patients and their principal caregivers. Canadian Medical Association Journal, 170(12), 1795-1801.

Hammeleft, K.J. (2015). Anxiety dans Carlton G. Brown. A guide to oncology symptom management (2nd ed., chap. 4, 55-76). Pittsburgh, PE; ONS.

Howard-Anderson, J., Ganz, P.A, Bower, J.E. et Stanton, A.L. (2012). Quality of life, fertility concerns and behavioral health outcomes in younger breast cancer survivors: A systematic review. Journal of National Cancer Institute, 104(5), 386-405.

Howell D., Currie S., Mayo S., Jones G., Boyle M., Hack T., et al. A Pan-Canadian clinical practice guideline: Assessment of psychosocial health care needs of the adult cancer patient. Toronto: Canadian Partnership Against Cancer (Cancer Journey Action Group) and the Canadian Association of Psychosocial Oncology; mai 2009.

Mitchell, A.J. (2007). Pooled results from 38 analyses of the accuracy of distress thermometer and other ultra-short methods of detecting cancer-related mood disorders. Journal of Clinical Oncology, 25(29), 4670-4681.

Mitchell, A.J. (2013). Depression and anxiety in long-term cancer survivors compared with spouses and healthy controls; a systematic review and meta-analysis. The Lancet Oncology, 14(8), 721-732. 
Mitchell, A.J., Hussain, N., Grainger, L. et Symonds, P. (2011). Identification of patient-reported distress by clinical nurse specialist in routine oncology practice: A multicentre UK study. Psycho-oncology, 20, 1076-1083.

National Comprehensive Cancer Network (NCCN) (2012). Clinical practice guidelines in oncology. Consulté sur https://www.nccn.org/ professionals/physician_gls/f_guidelines.asp

Niess, D. et Duffy, K.M. (2004). Basic concepts of transplantation. Dans S. Ezzone. Hematopoïtic stem cell transplantation. A manual for nursing practice. Pittsburgh, PA: Oncology Nursing Society.

Partenariat canadien contre le cancer (2009). Dépistage de la détresse, le $6^{e}$ signe vital : guide d'adoption de pratiques exemplaires pour des soins centrés sur la personne. Partie A : Contexte, recommandations et intégration [copie de travail]. Consulté sur http://www. partnershipagainstcancer.ca/wp-content/uploads/2.4.0.1.4.5Guide_CJAG.pdf

Polit, D.F. (2010). Statistics and Data Analysis and Statistics for Nursing Research (2 éd). New Jersey: Pearson Education Inc.

Programme Qmentum, Services de traitement du cancer et oncologie. (2010). Agrément Canada : Ottawa. http://www.accreditation.ca/ $\mathrm{fr} /$ content.aspx? pageid=50\&langType $=3084$.

Rusiewicz, A., DuHamel, K.N., Burkhalter, J., Ostroff, J.,Winkel, G.,Scigliano, E. et al. (2008). Psychological distress in long-term survivors of hematopoietic stem cell transplantation. Psycho-Oncology, 17(4), 329-337.
Salmon, P., Clark, L., McGrath, E. et Fisher, P. (2014). Screening for psychological distress in cancer; renewing the research agenda. Psycho-Oncology. doi:10.1002/pon.3640

Schofield, T., Connell, R., Walker, L. Wood, J., Butland, D. (2000). Understanding men's health and illness: A gender relations approach to policy research and practice. Journal of American College Health, 48(6), 247-256.

Société canadienne du cancer (2013). www.cancer.ca, Statistiques canadiennes sur le cancer. Page consultée en septembre 2013.

Suchocka-Capuano, A. et Bungener, C. (2010). Peur de la récidive et/ ou progression du cancer et prévention en santé mentale. PsychoOncologie. 4, 237-243.

Sun, C.-L., Francisco. L., Baker, S. Weisdorf, D.J., Forman, S.J. et Bathia, S. (2011). Blood, 118(17), 4723-4731.

Trask, P.C., Paterson, A., Riba, M., Brines, B., Grifith, K., Parker et al. (2002). Assessment of psychological distress in prospective bone marrow transplant patients. Bone Marrow Transplantation, 29, 917-925.

Vachon, M. (2006). Psychosocial distress and coping after cancer treatment. Cancer Nursing, 29(2 Suppl.), 26-31.

Zabora, J., Brintzenhofeszoc, K., Curbow, B., Hooker, C. et Piantadosi, S. (2001). The prevalence of psychological distress by cancer site. Psychooncology, 19(1), 19-28. 